\title{
Prognostic and predictive significance of podocalyxin-like protein expression in pancreatic and periampullary adenocarcinoma
}

Margareta Heby, Jakob Elebro, Björn Nodin, Karin Jirström and Jakob Eberhard*

\begin{abstract}
Background: Adenocarcinoma of the periampullary region is associated with poor prognosis and new prognostic and treatment predictive biomarkers are needed for improved treatment. Membranous expression of podocalyxin-like 1(PODXL), which is a cell-adhesion glycoprotein and stem cell marker, has been found to correlate with an aggressive tumour phenotype and adverse outcome in several cancer types. The aim of the present study was to examine the clinicopathological correlates, prognostic and predictive significance of tumour-specific PODXL expression in a retrospective cohort of pancreatic and periampullary carcinoma, morphologically divided into intestinal type (I-type) and pancreatobiliary type (PB-type) tumours.

Methods: Immunohistochemical expression of PODXL was analysed in tissue microarrays with primary tumours and a subset of paired lymph node metastases from 175 patients operated with pancreaticoduodenectomy for periampullary adenocarcinoma. Chi square test was applied to analyse the relationship between PODXL expression and clinicopathological parameters. Kaplan Meier analysis and Cox regression models were applied to estimate differences in 5-year overall survival (OS) and recurrence-free survival (RFS) in strata according to membranous and non-membranous PODXL expression.
\end{abstract}

Results: Membranous PODXL expression was significantly higher in primary PB-type (49.5 \%) as compared with I-type $(17.5 \%)$ tumours. In PB-type tumours, PODXL expression was significantly associated with female sex $(p=0.005)$, location to the pancreas $(p=0.005)$, and poor differentiation grade $(p=0.044)$. Membranous PODXL expression was significantly associated with a reduced RFS ( $H R=2.44,95 \% \mathrm{Cl} 1.10-5.44)$ and OS ( $H R=2.32,95 \% \mathrm{Cl} 1.05-5.12)$ in l-type tumours and with a reduced RFS ( $\mathrm{HR}=1.63,95 \% \mathrm{Cl} 1.07-2.49)$ but not OS in PB-type tumours. PODXL remained a significant independent prognostic factor only in l-type tumours ( $H R=5.12,95 \% \mathrm{Cl} 1.43-18.31$ for $\mathrm{RFS}$ and $\mathrm{HR}=7.31,95 \%$ Cl 2.12-25.16 for OS). Patients with I-type tumours displaying membranous PODXL expression had a significant beneficial effect of adjuvant chemotherapy regarding 5-year OS.

Conclusion: Membranous expression of PODXL is significantly higher in PB-type than in I-type periampullary adenocarcinomas and an independent factor of poor prognosis in the latter. The results further indicate a beneficial effect of adjuvant chemotherapy on I-type tumours with membranous PODXL expression, suggesting the potential utility of PODXL as a biomarker for improved treatment stratification of these patients.

Keywords: Periampullary adenocarcinoma, Pancreatic cancer, Podocalyxin-like 1, Immunohistochemistry, Biomarkers, Prognosis, Response prediction

\footnotetext{
* Correspondence: jakob.eberhard@med.lu.se

Department of Clinical Sciences Lund, Division of Oncology and Pathology,

Lund University, Skåne University Hospital, 22185 Lund, Sweden
} 


\section{Background}

Adenocarcinoma of the periampullary region, including tumours originating in the distal bile duct, pancreas, ampulla of Vater and the periampullary duodenum, are a heterogeneous group of neoplasms and despite advances in surgery, radiotherapy, chemotherapy and targeted agents, patients still suffer from a poor prognosis. The incidence of these tumours has markedly increased over the past decades and in 2012 pancreatic cancers of all types were the seventh most common cause of cancer deaths, resulting in 330.000 deaths globally [1]. The overall 5 -year survival is $5 \%$, all stages of the disease combined, and the median survival has been reported to be $5-8$ months [2-4]. There are no early detection tests and most patients with localized disease have no recognizable symptoms or signs, resulting in late diagnosis in the majority of cases. Only15$20 \%$ of the tumours are resectable at presentation [5], resectability often being limited by early local invasion of the surrounding anatomical structures, such as mesenteric arteries, or distant metastasis. There are two major morphological types of periampullary adenocarcinomas, i.e. pancreatobiliary (PB-type) adenocarcinomas (including pancreatic cancer, distal bile duct cancer, and some of the ampullary carcinomas) and intestinal type (I-type) periampullary adenocarcinomas (including duodenal carcinoma and some of the ampullary carcinomas). Morphological type seems to provide more important prognostic information in resected periampullary carcinoma than the tumour origin, with PB-type tumours being associated with significantly shorter survival rates than I-type tumours $[6,7]$. The present diagnostic and prognostic information provided by histopathological parameters is far from sufficient, strongly implicating the need for additional molecular-based biomarkers to better define clinically relevant subgroups of these tumours for improved treatment stratification.

Podocalyxin-like protein (PODXL) is a member of the CD34 family of transmembrane sialomucins. PODXL is expressed on the apical surface of glomerular epithelial cells and podocytes [8], where it plays an integral role in maintaining adequate filtration [9], and it is also expressed on vascular endothelia [10] and hematopoietic stem cells $[11,12]$. PODXL is upregulated in several types of cancer, and strong expression, in particular in the cell membrane, has been demonstrated to signify more aggressive tumours and poor prognosis in e.g. breast cancer [13], colorectal cancer [14-17] ovarian cancer [18], urinary bladder cancer [19], and glioblastoma [20].

PODXL has been found to be more frequently expressed (44 \%) in pancreatic ductal adenocarcinoma as compared with other types of adenocarcinomas of the gastrointestinal and biliary tracts [21]. In another study, sialofucosylated PODXL was demonstrated to be a functional E- and Lselectin ligand expressed by metastatic pancreatic cancer cells in vitro, and was also found to be overexpressed, with membranous localization, in $69 \%$ of 105 pancreatic ductal adenocarcinomas [22]. To our knowledge, the prognostic or predictive impact of PODXL expression in pancreatic or periampullary adenocarcinoma has not yet been described. The aim of the present study was therefore to examine the clinicopathological correlates, prognostic and predictive significance of tumour-specific PODXL expression in a retrospective cohort of pancreatic and periampullary adenocarcinoma, with particular reference to morphological subtypes thereof.

\section{Methods \\ Patients}

The study consists of a retrospective consecutive cohort of 175 patients with primary periampullary adenocarcinomas, surgically treated with pancreaticoduodenectomy at the University hospitals of Lund and Malmö, Sweden, from January 12001 until December 312011 [23-25]. Out of 175 cases in the entire cohort, there were 110 pancreatobiliary-type and 65 intestinal-type adenocarcinomas. Survival data were collected from the Swedish National Civil Register. Follow-up started at the date of surgery and ended at death, at 5 years after surgery or at December 31, 2013, whichever came first. Information on neoadjuvant and adjuvant treatment and recurrence was obtained from patient records.

All haematoxylin \& eosin stained slides from all cases were re-evaluated by one pathologist (JEL), blinded to the original report and outcome. The decision on tumour origin and morphological type was based on several criteria, as previously described [23].

The study has been approved by the Ethics Committee of Lund University ( $\mathrm{ref} \mathrm{nr} 445 / 07$ ).

\section{Tissue microarray construction}

Tissue microarrays (TMAs) were constructed using a semi-automated arraying device (TMArrayer, Pathology Devices, Westminister, MD, USA). A standard set of three tissue cores $(1 \mathrm{~mm})$ were obtained from each of the 175 primary tumours and from lymph node metastases from 105 of the cases, whereby one to three lymph node metastases were sampled in each case. Paired samples with non-malignant pancreatic tissue from the resection specimens were also obtained from 50 of the cases, using a standard set of two $1 \mathrm{~mm}$ tissue cores.

\section{Immunohistochemistry and staining evaluation}

For immunohistochemical analysis of PODXL expression, $4 \mu \mathrm{m}$ TMA-sections were automatically pre-treated using the PT Link system and then stained in an Autostainer Plus (DAKO; Glostrup, Copenhagen, Denmark) with the affinity-purified polyclonal, monospecific PODXL antibody (HPA002110; Atlas Antibodies AB, Stockholm, 
Sweden) diluted 1: 250. This antibody, originally generated within the Human Protein Atlas (HPA) project, has also been used in and validated in several previous biomarker studies on e.g. colorectal, bladder, pancreatic and testicular cancer $[14,19,22,26]$. The expression of PODXL was recorded as negative (0), weak cytoplasmic positivity in any proportion of cells (1), moderate cytoplasmic positivity in any proportion of cells (2), distinct membranous positivity in $<=50 \%$ of cells (3) and distinct membranous positivity in $>50 \%$ of cells (4) as previously described [14-16, 19]. Staining of PODXL was evaluated by two independent observers ( $\mathrm{MH}$ and $\mathrm{KJ})$ who were blinded to clinical and outcome data. Scoring differences were discussed in order to reach consensus.

\section{Statistical analysis}

Chi square test was applied to analyse the relationship between PODXL expression and clinicopathological parameters. Two patients with $\mathrm{PB}$-type adenocarcinomas who had received neoadjuvant chemotherapy were excluded from the correlation and survival analyses. Three additional patients were excluded from the survival analyses; two with I-type adenocarcinomas who died within one month from surgery due to complications and one with PB-type adenocarcinoma who emigrated 5 months after surgery.

Kaplan Meier analysis and log rank test were applied to estimate differences in 5-year overall survival (OS) and recurrence-free survival (RFS) in strata according to membranous and non-membranous PODXL expression. Hazard ratios (HR) for death and recurrence within 5 years were calculated by Cox regression proportional hazard's modelling in unadjusted analysis and in a multivariable model adjusted for age, sex, T-stage, N-stage, differentiation grade, lymphatic invasion, vascular invasion, perineural invasion, infiltration in peripancreatic fat, resection margins, tumour origin, and adjuvant chemotherapy. A backward conditional method was used for variable selection in the adjusted model. To estimate the interaction effect between adjuvant treatment and PODXL expression in order to measure any possible difference in treatment effect based on PODXL expression, the following interaction variable was constructed; any adjuvant treatment $(+/-) \times$ PODXL $(+/-)$.

All tests were two sided. P-values $<0.05$ were considered significant. All statistical analyses were performed using IBM SPSS Statistics version 20.0 (SPSS Inc., Chicago, IL, USA).

\section{Results}

PODXL expression in non-malignant pancreas, primary tumours and lymph node metastases

Sample immunohistochemical images of PODXL expression are shown in Fig. 1. PODXL expression could be assessed in in 63/65 (96.9\%) primary I-type carcinomas and $24 / 30(80.0 \%)$ lymph node metastases, and in 107/ 108 (99.1\%) primary PB-type carcinomas and 63/75 (84.0 \%) corresponding lymph node metastases. PODXL expression could be assessed in 49/50 (98 \%) paired non-malignant samples, all displaying negative or very weak PODXL expression in acini and ducts. The distribution of PODXL expression in primary tumours and metastases, which did not differ significantly, by histological subtype, is shown in Fig. 2. Membranous PODXL expression was denoted in 11/63 (17.5 \%) primary and $2 / 24(8.3 \%)$ metastatic I-type carcinomas, and in 53/107 (49.5\%) primary and 23/63 (36.5\%) metastatic PB-type carcinomas. In I-type tumours, membranous PODXL in the metastasis was seen in 1/21 (4.8\%) cases denoted as having non-membranous expression in the primary tumour, and non-membranous PODXL expression in the metastasis was denoted in 2/3 (66.7 \%) cases with primary tumours displaying membranous PODXL expression. In PB-type tumours, the number of cases with non-membranous to membranous conversion was 2/32 (6.2 \%) and with membranous to non-membranous conversion 10/31 (32.3\%). In all further statistical analyses, a dichotomized variable of non-membranous (score $0,1,2$ ) versus membranous (score $3,4)$ PODXL expression in the primary and/or metastatic component is applied. According to this combined variable, 12/63 (19.0 \%) I-type cases and 55/107 (51.4 \%) PB-type cases displayed membranous PODXL expression in any component.

\section{Associations of PODXL expression with clinicopathological factors}

The associations between PODXL expression and clinicopathological factors in I-type and PB-type tumours, respectively, are shown in Table 1. In I-type tumours, there were no significant associations between PODXL expression and clinicopathological factors. In PB-type tumours, membranous PODXL expression was significantly associated with female sex $(p=0.005)$, with location to the pancreas $(p=0.005)$, and with poor differentiation grade $(p=0.044)$. There was no statistically significant association between PODXL expression and other clinicopathological factors including age at diagnosis, tumour size, T-stage, $\mathrm{N}$ stage, resection margins, presence of vascular- lymphatic and neural invasion and growth in peripancreatic fat.

\section{Prognostic and potential predictive value of PODXL expression}

As demonstrated in Fig. 3, Kaplan-Meier analysis revealed significant associations of membranous PODXL expression with a reduced RFS (logrank $p=0.024)$ and OS (logrank $p=0.032$ ) in I-type tumours and with a reduced RFS (logrank $p=0.022$ ) but not OS in PB-type tumours. These associations were confirmed in univariable Cox regression 


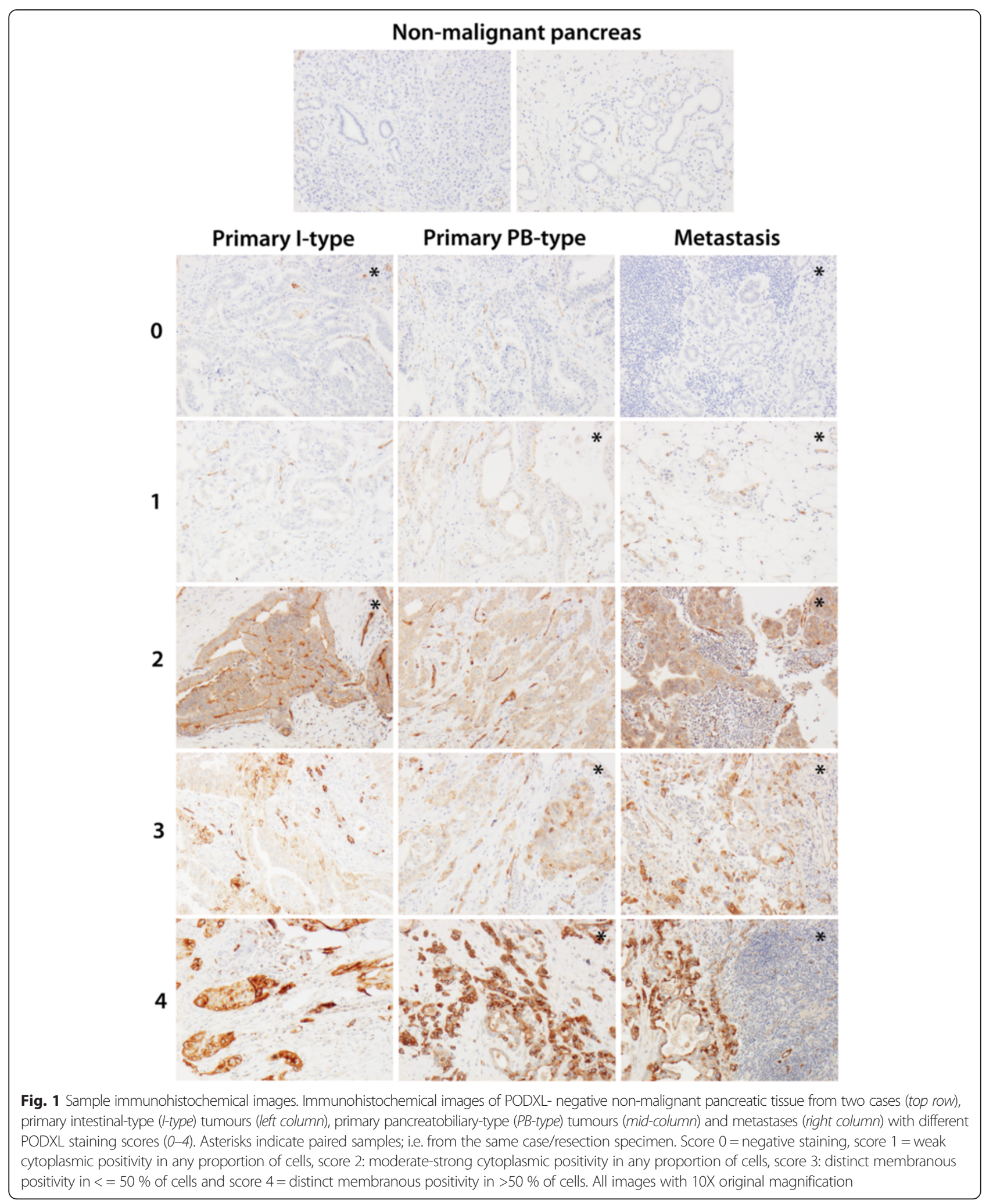

analysis for both RFS (Table 2) and OS (Table 3) in I-type tumours $(\mathrm{HR}=2.44,95 \% \mathrm{CI} 1.10-5.44$, and $\mathrm{HR}=2.32$, $95 \%$ CI 1.05-5.12, respectively) and for RFS (Table 2), but not OS (Table 3) in PB-type tumours (HR $=1.63,95 \% \mathrm{CI}$ 1.07-2.49, logrank $p=0.022$ ). In multivariable analysis, PODXL remained a significant prognostic factor only in 

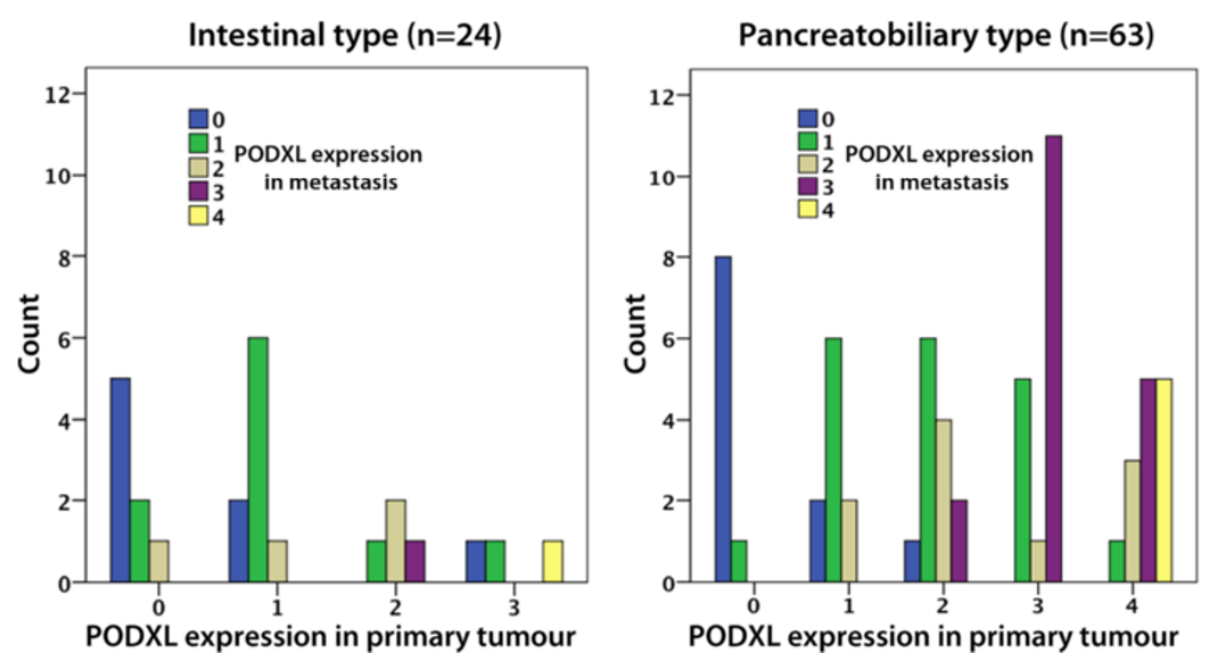

Fig. 2 PODXL expression in primary tumours and metastases

I-type tumours $(\mathrm{HR}=5.12,95 \% \mathrm{CI} 1.43-18.31$ for RFS, Table 2, and HR = 7.31, $95 \%$ CI 2.12-25.16 for OS, Table 3).

Next, we examined the potential predictive impact of PODXL expression on survival in strata according to adjuvant treatment. As demonstrated in Fig. 4, patients with I-type tumours displaying membranous PODXL expression had a significant beneficial effect of adjuvant chemotherapy regarding 5-year OS. When ampullary PBtype tumours, expressing membranous PODXL in a similar proportion to I-type tumours, were included in the analysis, the beneficial value of adjuvant chemotherapy was even more pronounced (Fig. 4). Hazard ratios for 5year OS according to adjuvant treatment and PODXL expression are shown in Additional file 1. The results demonstrate that survival did not differ significantly by membranous PODXL-expression in patients with I-type tumours or the extended group of I-type + ampullary PBtype tumours having received adjuvant chemotherapy. In contrast, the adverse prognostic impact of membranous PODXL expression was even more evident in patients not receiving adjuvant chemotherapy compared to the entire group (unadjusted $\mathrm{HR}=4.38,95 \%$ CI 1.57-12.18 in I-type tumours, and unadjusted $\mathrm{HR}=7.13$; $95 \% \mathrm{CI} 2.64-19.26$ in I-type + ampullary PB-type tumours). These associations remained significant in multivariable analysis, but there was no significant treatment interaction (Additional file 1). These associations were not significant in relation to RFS (data not shown) or in PB-type tumours (data not shown). The prognostic and predictive impact of membranous PODXL expression was similar when only its expression in the primary tumour was considered (data not shown). The prognostic value of the full range of PODXL scores (0-4) in relation to RFS and OS, in the entire cohort and by morphological subtype, is shown in Additional file 2. All survival analyses were also performed using a dichotomized variable of score $0-1$ vs $2-4$, with allover less significant results (data not shown).

\section{Discussion}

Pancreatic cancer is an extremely lethal type of cancer. On average, patients die from the disease within 6 months from diagnosis. Therefore it is of uttermost importance to find both predictive and prognostic factors so as to improve treatment. The results from this study provide a first demonstration of the prognostic and potential predictive value of PODXL in pancreatic, distal bile duct, ampullary and duodenal adenocarcinoma. PODXL-expression was found to be significantly higher in PB-type as compared with I-type tumours, with the exception for ampullary PBtype tumours. These findings are in line with the expected and provide further evidence of PODXL being associated with a more aggressive tumour phenotype and a biomarker of poor prognosis in human cancer.

The study cohort encompasses a retrospective cohort of 110 pancreatobiliary-type and 65 intestinal-type adenocarcinomas, including paired normal tissue and lymph node metastases from a subset of cases, thus providing a thorough characterization of PODXL expression in a wide range of periampullary adenocarcinomas. In the present study, membranous PODXL expression was denoted in $49.5 \%$ of primary PB-type carcinomas, which is somewhat lower than in the previous study by Dallas et al., including tumours from 105 cases assembled in TMAs, wherein membranous PODXL expression was found in $69 \%$ of the cases [22]. In primary I-type carcinomas, membranous PODXL expression was denoted in $17.5 \%$, which is well in line with previous TMA-based studies on colorectal cancer wherein membranous expression was found in $13.4 \%$ and $9.6 \%$ respectively $[14,15]$. This observation further supports the theory that I-type carcinomas of the 
Table 1 Associations between membranous and non-membranous PODXL expression with clinciopathological parameters in intestinal type and pancreatobiliary type tumours, respectively

\begin{tabular}{|c|c|c|c|c|c|c|}
\hline \multirow{3}{*}{. } & \multicolumn{3}{|l|}{ Intestinal type } & \multicolumn{3}{|c|}{ Pancreatobiliary type } \\
\hline & PODXL NM & PODXL M & $P$ & PODXL NM & PODXL M & $P$ \\
\hline & $(n=51)$ & $(n=12)$ & & $(n=52)$ & $(n=55)$ & \\
\hline \multicolumn{7}{|l|}{ Age } \\
\hline (median, range) & $66.0(38.0-83.0)$ & $67.5(44.0-74.0)$ & 0.972 & $66.0(44.0-81.0)$ & $68.0(44.0-81.0)$ & 0.613 \\
\hline \multicolumn{7}{|l|}{ Sex } \\
\hline Women & $28(82.4)$ & $6(17.6)$ & 0.761 & $17(34.0)$ & $33(66.0)$ & 0.005 \\
\hline Men & $23(79.3)$ & $6(20.7)$ & & $35(61.4)$ & $22(38.6)$ & \\
\hline \multicolumn{7}{|l|}{ Tumour origin } \\
\hline Duodenum & $12(85.7)$ & $2(14.3)$ & 0.610 & & & \\
\hline Ampulla intestinal type & $39(79.6)$ & $10(20.4)$ & & & & \\
\hline Ampulla pancreatobiliary type & & & & $14(73.7)$ & $5(26.3)$ & 0.005 \\
\hline Distal bile duct & & & & $23(51.1)$ & $22(48.9)$ & \\
\hline Pancreas & & & & $15(34.9)$ & $28(65.1)$ & \\
\hline \multicolumn{7}{|l|}{ Tumour size $\mathrm{mm}$} \\
\hline (median, range) & $30.0(5.0-90.0)$ & $26.5(12.0-40.0)$ & 0.923 & $30.0(5.0-70.0)$ & $30.0(15.0-70.0)$ & 0.313 \\
\hline \multicolumn{7}{|l|}{ Differentiation grade } \\
\hline Well-moderate & $26(83.9)$ & $5(16.1)$ & 0.565 & $24(61.5)$ & $15(38.5)$ & 0.044 \\
\hline Poor & $25(78.1)$ & $7(21.9)$ & & $28(41.2)$ & $40(58.8)$ & \\
\hline \multicolumn{7}{|l|}{ T-stage } \\
\hline $\mathrm{T} 1$ & $4(100.0)$ & $0(0.0)$ & 0.246 & $2(100.0)$ & $0(0.0)$ & 0.392 \\
\hline $\mathrm{T} 2$ & $9(81.8)$ & $2(18.2)$ & & $4(40.0)$ & $6(60.0)$ & \\
\hline $\mathrm{T} 3$ & $21(84.0)$ & $4(16.0)$ & & $34(43.6)$ & $44(56.4)$ & \\
\hline T4 & $17(73.9)$ & $6(26.1)$ & & $12(70.6)$ & $5(29.4)$ & \\
\hline \multicolumn{7}{|l|}{ N-stage } \\
\hline No & $28(84.8)$ & $5(5.2)$ & 0.936 & $17(56.7)$ & $13(43.3)$ & 0.315 \\
\hline N1 & $13(68.4)$ & $6(31.6)$ & & $21(46.7)$ & $24(53.3)$ & \\
\hline $\mathrm{N} 2$ & $10(90.9)$ & $1(9.1)$ & & $14(43.8)$ & $18(56.2)$ & \\
\hline \multicolumn{7}{|l|}{ Margins } \\
\hline RO & $15(88.2)$ & $2(11.8)$ & 0.375 & $3(50.0)$ & $3(50.0)$ & 0.944 \\
\hline$R 1-R x$ & $36(78.3)$ & $10(21.7)$ & & $49(48.5)$ & $52(51.5)$ & \\
\hline \multicolumn{7}{|l|}{ Perineural growth } \\
\hline No & $38(86.4)$ & $6(13.6)$ & 0.099 & $14(63.6)$ & $8(36.4)$ & 0.115 \\
\hline Yes & $13(68.4)$ & $6(31.6)$ & & $38(44.7)$ & $47(55.3)$ & \\
\hline \multicolumn{7}{|l|}{ Invasion of lymphatic vessels } \\
\hline No & $26(89.7)$ & $3(10.3)$ & 0.107 & $16(50.0)$ & $16(50.0)$ & 0.850 \\
\hline Yes & $25(73.5)$ & $9(26.5)$ & & $36(48.0)$ & $39(52.0)$ & \\
\hline \multicolumn{7}{|l|}{ Invasion of blood vessels } \\
\hline No & $48(82.8)$ & $10(17.2)$ & 0.217 & $35(50.0)$ & $35(50.0)$ & 0.691 \\
\hline Yes & $3(60.0)$ & $2(40.0)$ & & $17(45.9)$ & $20(54.1)$ & \\
\hline \multicolumn{7}{|l|}{ Growth in peripancreatic fat } \\
\hline No & $35(85.4)$ & $6(14.6)$ & 0.227 & $13(59.1)$ & $9(40.9)$ & 0.271 \\
\hline Yes & $16(72.7)$ & $6(27.3)$ & & $39(45.9)$ & $46(54.1)$ & \\
\hline
\end{tabular}


Table 1 Associations between membranous and non-membranous PODXL expression with clinciopathological parameters in intestinal type and pancreatobiliary type tumours, respectively (Continued)

\begin{tabular}{|c|c|c|c|c|c|c|}
\hline \multicolumn{7}{|l|}{ Adjuvant chemotherapy } \\
\hline None & $40(88.9)$ & $5(11.1)$ & 0.145 & $23(46.0)$ & $27(54.0)$ & 0.348 \\
\hline 5-FU-analogue & $2(40.0)$ & $3(60.0)$ & & $5(62.5)$ & $3(37.5)$ & \\
\hline Gemcitabine & $4(57.1)$ & $3(42.9)$ & & $20(45.5)$ & $24(54.5)$ & \\
\hline Gemcitabine + capecitabine & $1(100.0)$ & $0(0.0)$ & & $1(50.0)$ & $1(50.0)$ & \\
\hline Oxaliplatin + 5-FU analogue & $4(100.0)$ & $0(0.0)$ & & $1(100.0)$ & $0(0.0)$ & \\
\hline Gemcitabine + oxaliplatin & $0(0.0)$ & $1(100.0)$ & & $2(100.0)$ & $0(0.0)$ & \\
\hline
\end{tabular}

$M$ membranous PODXL expression, NM non-membranous PODXL expression

$R 0$ radical resection, $R 1$ non-radical resection, $R X$ uncertain resection margins

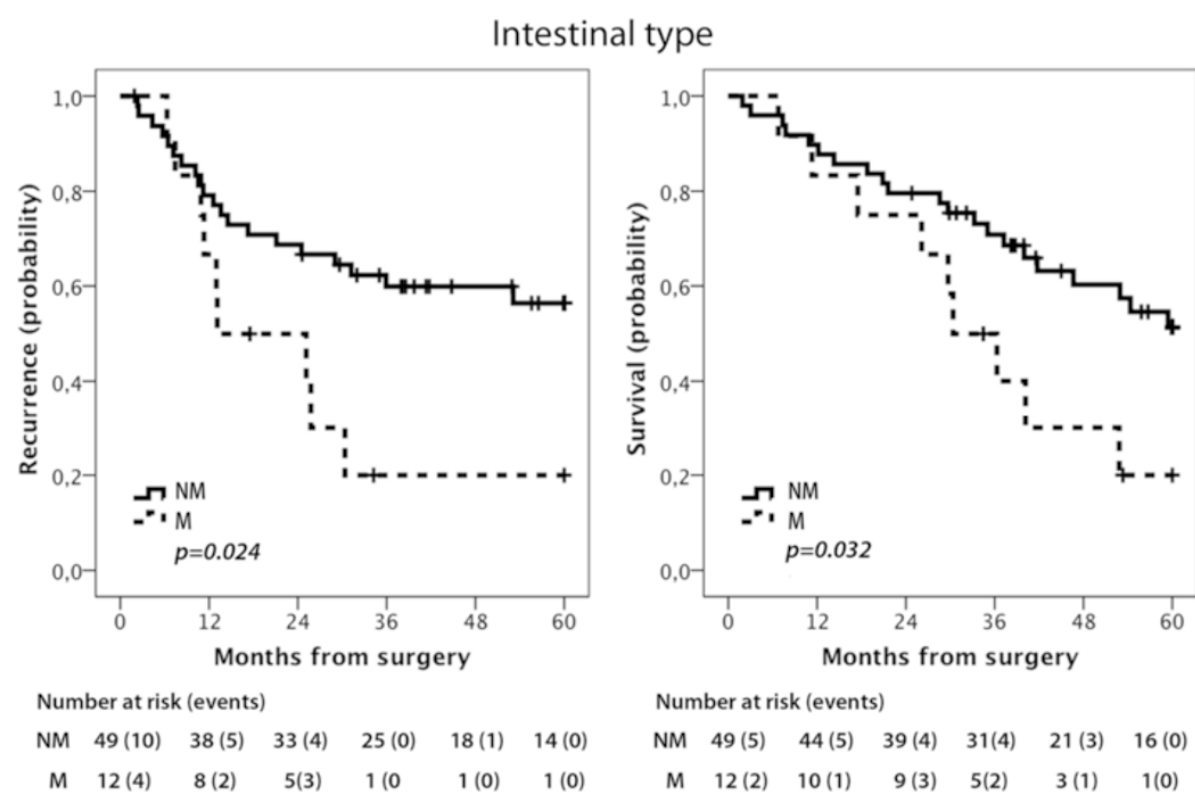

Pancreatobiliary type

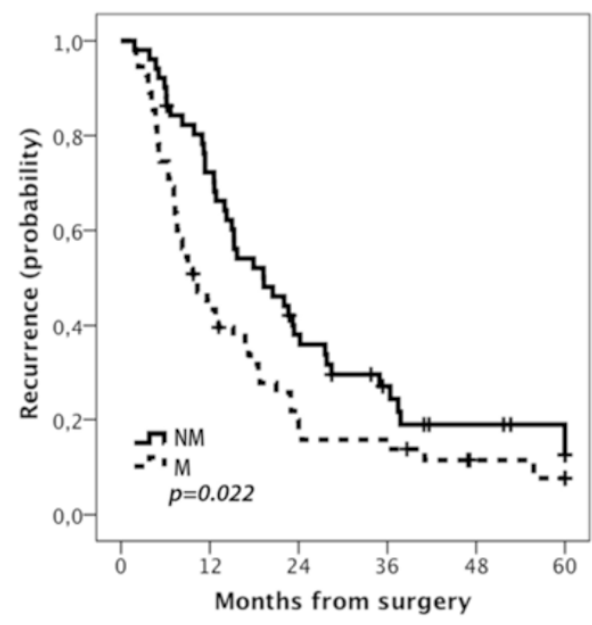

Number at risk (events)

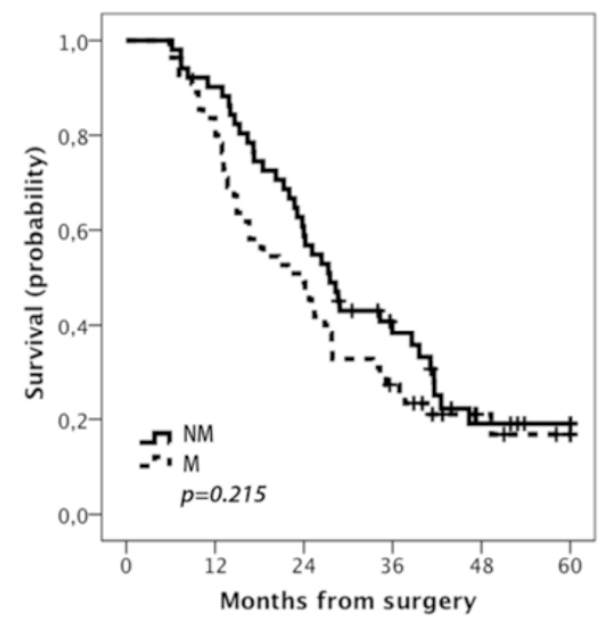

NM $\quad 51(14) \quad 36(17) \quad 18(5) \quad 10(3) \quad(5(0) \quad 3(1)$

Number at risk (events)

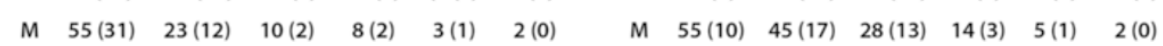

Fig. 3 Survival according to membranous versus non-membranous PODXL expression. Kaplan-Meier estimates of recurrence free and 5-year overall survival in patients with intestinal and pancreatobiliary type tumours, respectively. $\mathrm{NM}=$ non-membranous, $\mathrm{M}=$ membranous PODXL expression 
Table 2 Unadjusted and adjusted hazard ratios for recurrence within five years in intestinal and pancreatobiliary type tumours

\begin{tabular}{|c|c|c|c|c|c|c|}
\hline & \multicolumn{3}{|c|}{ Intestinal type } & \multicolumn{3}{|c|}{ Pancreatobiliary type } \\
\hline & & Unadjusted & Adjusted & & Unadjusted & Adjusted \\
\hline & n(events) & $\mathrm{HR}(95 \% \mathrm{Cl})$ & $\mathrm{HR}(95 \% \mathrm{Cl})$ & n(events) & $\mathrm{HR}(95 \% \mathrm{Cl})$ & $\mathrm{HR}(95 \% \mathrm{Cl})$ \\
\hline \multicolumn{7}{|l|}{ Age } \\
\hline Continuous & $61(29)$ & $1.00(0.96-1.03)$ & $1.07(1.02-1.13)$ & $106(88)$ & $0.98(0.96-1.01)$ & $1.00(0.96-1.03)$ \\
\hline \multicolumn{7}{|l|}{ Gender } \\
\hline Female & $34(11)$ & 1.00 & 1.00 & $50(42)$ & 1.00 & 1.00 \\
\hline Male & $27(18)$ & $2.31(1.08-4.94)$ & $2.40(0.94-6.14)$ & $56(46)$ & $1.06(0.70-1.61)$ & $0.88(0.53-1.49)$ \\
\hline \multicolumn{7}{|l|}{ Tumour origin } \\
\hline Duodenum & $13(4)$ & 1.00 & 1.00 & & - & - \\
\hline Ampulla-Intestinal type & $48(25)$ & $2.18(0.76-6.27)$ & $6.82(1.32-35.12)$ & & - & - \\
\hline Ampulla-Pancreatobiliary type & & - & - & $19(16)$ & 1.00 & 1.00 \\
\hline Distal Bile duct & & - & - & $44(38)$ & $1.10(0.61-1.98)$ & $1.52(0.78-2.94)$ \\
\hline Pancreas & & - & - & $43(34)$ & $1.06(0.58-1.93)$ & $0.93(0.49-1.77)$ \\
\hline \multicolumn{7}{|l|}{ Tumour size } \\
\hline Continuous & $61(30)$ & $1.00(0.98-1.02)$ & $1.04(1.00-1.09)$ & $106(88)$ & $1.03(1.02-1.05)$ & $1.02(0.99-1.04)$ \\
\hline \multicolumn{7}{|l|}{ T-stage } \\
\hline $\mathrm{T} 1$ & $4(1)$ & 1.00 & 1.00 & $2(1)$ & 1.00 & 1.00 \\
\hline $\mathrm{T} 2$ & 10(3) & $1.28(0.13-12.30)$ & $3.71(0.34-40.64)$ & $10(6)$ & $1.61(0.19-13.36)$ & $0.66(0.07-6.08)$ \\
\hline T3 & $25(9)$ & $1.86(0.24-14.71)$ & $5.72(0.50-65.06)$ & $77(66)$ & $4.67(0.64-33.96)$ & $1.21(0.15-9.92)$ \\
\hline T4 & $22(16)$ & $5.44(0.72-41.21)$ & $6.36(0.21-195.20)$ & $17(15)$ & $4.31(0.56-33.10)$ & $1.83(0.08-40.27)$ \\
\hline \multicolumn{7}{|l|}{ N-stage } \\
\hline No & $33(11)$ & 1.00 & 1.00 & $29(21)$ & 1.00 & 1.00 \\
\hline N1 (metastasis in 1-3 |gl) & $19(11)$ & $2.07(0.90-4.78)$ & $1.00(0.37-2.70)$ & $45(37)$ & $2.17(1.25-3.78)$ & $2.04(1.13-3.67)$ \\
\hline N2 (metastasis in 4 or more lgl) & $9(7)$ & $4.06(1.55-10.59)$ & $6.88(1.81-26.15)$ & $32(30)$ & $3.11(1.72-5.61)$ & $2.61(1.42-4.83)$ \\
\hline \multicolumn{7}{|l|}{ Differentiation grade } \\
\hline Well-moderate & $30(11)$ & 1.00 & 1.00 & $39(29)$ & 1.00 & 1.00 \\
\hline Poor & $31(18)$ & $2.16(1.02-4.57)$ & $1.38(0.40-4.79)$ & $67(59)$ & $2.32(1.45-3.71)$ & $2.02(1.20-3.39)$ \\
\hline \multicolumn{7}{|l|}{ Involved margins, status } \\
\hline RO & $17(3)$ & 1.00 & 1.00 & $6(4)$ & 1.00 & 1.00 \\
\hline$R 1 \& R x$ & $44(26)$ & $4.51(1.36-14.94)$ & $2.23(0.62-8.03)$ & $100(84)$ & $2.31(0.84-6.36)$ & $2.39(0.84-6.76)$ \\
\hline \multicolumn{7}{|l|}{ Lymphatic growth } \\
\hline Absent & $28(5)$ & 1.00 & 1.00 & $32(23)$ & 1.00 & 1.00 \\
\hline Present & $33(24)$ & $6.16(2.34-16.19)$ & $6.19(1.76-21.82)$ & $74(65)$ & $1.77(1.09-2.88)$ & $1.05(0.59-1.85)$ \\
\hline \multicolumn{7}{|l|}{ Vascular growth } \\
\hline Absent & $56(24)$ & 1.00 & 1.00 & $70(55)$ & 1.00 & 1.00 \\
\hline Present & $5(5)$ & $8.16(2.86-23.30)$ & $1.62(0.39-6.65)$ & $36(33)$ & $2.30(1.47-3.61)$ & $2.08(1.28-3.36)$ \\
\hline \multicolumn{7}{|l|}{ Perineural growth } \\
\hline Absent & $42(15)$ & 1.00 & 1.00 & $22(14)$ & 1.00 & 1.00 \\
\hline Present & $19(14)$ & $2.72(1.31-5.66)$ & $1.01(0.27-3.81)$ & $84(74)$ & $2.93(1.57-5.46)$ & $2.04(1.06-3.90)$ \\
\hline \multicolumn{7}{|l|}{ Growth in peripancreatic fat } \\
\hline Absent & $40(12)$ & 1.00 & 1.00 & $22(13)$ & 1.00 & 1.00 \\
\hline Present & $21(17)$ & $4.74(2.23-10.10)$ & $3.60(1.43-9.07)$ & $84(75)$ & $2.60(1.42-4.75)$ & $1.45(0.76-2.77)$ \\
\hline
\end{tabular}


Table 2 Unadjusted and adjusted hazard ratios for recurrence within five years in intestinal and pancreatobiliary type tumours (Continued)

\begin{tabular}{|c|c|c|c|c|c|c|}
\hline \multicolumn{7}{|l|}{ Adjuvant treatment } \\
\hline No & $43(21)$ & 1.00 & 1.00 & $49(40)$ & 1.00 & 1.00 \\
\hline Yes & $18(8)$ & $0.87(0.38-1.96)$ & $0.12(0.04-0.44)$ & $57(48)$ & $1.08(0.70-1.65)$ & $0.89(0.54-1.49)$ \\
\hline \multicolumn{7}{|l|}{ PODXL expression } \\
\hline Non-membranous & $49(20)$ & 1.00 & 1.00 & $51(40)$ & 1.00 & 1.00 \\
\hline Membranous & $12(9)$ & $2.44(1.10-5.44)$ & $5.12(1.43-18.31)$ & $55(88)$ & $1.63(1.07-2.49)$ & $1.53(0.99-2.38)$ \\
\hline
\end{tabular}

$R 0$ radical resection, $R 1$ non-radical resection, $R X$ uncertain resection margins

pancreatic region resemble tumours with colorectal origin in a stronger way than PB-types. In line with the study by Ney et al., PODXL was negative or only weakly expressed in normal pancreatic parenchyma from the resection specimens [21].

In a previous study on colorectal cancer, wherein a PODXL expression was compared in full-face sections from 31 primary tumours and all available lymph node metastases $(n=140)$, there was an excellent concordance in that all primary tumours with non-membranous PODXL expression had metastases with non-membranous expression, whereas a few primary tumours with membranous PODXL expression had a varying proportion of metastatic lymph nodes with membranous and nonmembranous PODXL expression [16]. These findings led to the conclusion that for prognostic or predictive purposes, analysis of the primary tumour would be sufficient [16]. In the present study, although negative conversion of membranous PODXL expression from primary tumour to lymph node metastasis was far more common than positive conversion, a few cases displayed the latter phenomenon. Of note, all analyses were based on TMA-samples, and therefore, future studies based on full-face sections are warranted to further examine the rate of positive conversion of membranous PODXL expression in pancreatic and periampullary cancers, so as to determine whether biomarker analysis of the primary tumour will be sufficient in the clinical setting.

In the present study membranous PODXL expression was an independent predictor of reduced 5-year overall and recurrence-free survival in I-type but not in PB-type tumours, although there was a significant association between membranous PODXL expression and a reduced RFS in the latter in unadjusted analysis. These findings are well in line with previous publications regarding the prognostic significance of PODXL expression in several other major types of cancer [13-15, 18-20]. In addition, and importantly, we found that patients with I-type tumours displaying membranous PODXL had a beneficial effect of adjuvant chemotherapy. When ampullary PBtype tumours, expressing membranous PODXL in a similar proportion to I-type tumours, were included in the analysis, the effect by adjuvant chemotherapy was even more pronounced. This supports earlier data that patients with PODXL positive tumours benefit from adjuvant chemotherapy, irrespective of treatment regime, as seen in colorectal cancer [14, 15]. Moreover, these findings indicate that I-type tumours with high expression of PODXL are more likely to benefit from adjuvant therapy than PBtype tumours. Today, all patients with pancreatic and periampullary adenocarcinoma are recommended adjuvant treatment. Since adjuvant treatment often is associated with toxicity and adverse side effects, it is important to identify novel predictive and prognostic factors, such as PODXL, to support and improve clinical decisions. Therefore, the results from the present study indicate that PODXL could be used as a predictive marker for adjuvant treatment of periampullary cancer with intestinal morphology, and possibly also ampullary PB-type tumours. Of note, given the retrospective nature of the present study, the term "predictive" should be applied with caution. It must however be pointed out that the study cohort encompasses a consecutive series of clinically and histopathologically well-annotated pancreatoduodenectomy cases, of which only approximately half have been given adjuvant chemotherapy, which should allow for a fairly good assessment of both prognostic and predictive biomarkers even in the retrospective setting. Thus, the herein observed prognostic and potential predictive value of PODXL, in particular in I-type tumours, is of potential clinical relevance and merits further study in additional retrospective cohorts as well as in a controlled, prospective trial. Targeting PODXL with monoclonal antibodies may also be a future treatment option [27].

Membranous PODXL expression was considerably higher in PB-type as compared with I-type tumours, which is in line with the former being clinically more aggressive. In PB-type tumours, the prognostic value of PODXL was only significant for RFS, and not after adjustment for other clinicopathological factors, and there was no evident predictive value. The choice of prognostic cutoff, i.e. membranous vs non-membranous PODXL expression, can be considered appropriate for the herein used antibody, since the same antibody and cutoff has been used in the previous study on pancreatic cancer by Dallas et al. [22] and since this dichotomization yielded the strongest prognostic and predictive 
Table 3 Unadjusted and adjusted hazard ratios for death within five years in intestinal and pancreatobiliary type tumours

\begin{tabular}{|c|c|c|c|c|c|c|}
\hline & \multicolumn{3}{|c|}{ Intestinal type } & \multicolumn{3}{|c|}{ Pancreatobiliary type } \\
\hline & & Unadjusted & Adjusted & & Unadjusted & Adjusted \\
\hline & n(events) & $\mathrm{HR}(95 \% \mathrm{Cl})$ & $\mathrm{HR}(95 \% \mathrm{Cl})$ & n(events) & $\mathrm{HR}(95 \% \mathrm{Cl})$ & $\mathrm{HR}(95 \% \mathrm{Cl})$ \\
\hline \multicolumn{7}{|l|}{ Age } \\
\hline Continuous & $61(30)$ & $1.02(0.98-1.06)$ & $1.07(1.02-1.13)$ & $106(82)$ & $0.99(0.96-1.02)$ & $1.01(0.98-1.05)$ \\
\hline \multicolumn{7}{|l|}{ Gender } \\
\hline Female & $34(13)$ & 1.00 & 1.00 & $50(36)$ & 1.00 & 1.00 \\
\hline Male & $27(17)$ & $1.85(0.89-3.84)$ & $2.12(0.86-5.22)$ & $56(46)$ & $1.20(0.78-1.87)$ & $1.22(0.76-1.95)$ \\
\hline \multicolumn{7}{|l|}{ Tumour origin } \\
\hline Duodenum & $13(5)$ & 1.00 & 1.00 & & - & - \\
\hline Ampulla-Intestinal type & $48(25)$ & $1.49(0.57-3.88)$ & $7.77(1.86-32.39)$ & & - & - \\
\hline Ampulla-Pancreatobiliary type & & - & - & $19(16)$ & 1.00 & 1.00 \\
\hline Distal Bile duct & & - & - & $44(32)$ & $0.74(0.40-1.34)$ & $1.03(0.50-2.15)$ \\
\hline Pancreas & & - & - & $43(34)$ & $0.91(0.50-1.65)$ & $1.06(0.52-2.19)$ \\
\hline \multicolumn{7}{|l|}{ Tumour size } \\
\hline Continuous & $61(30)$ & $1.00(0.98-1.03)$ & $1.05(1.01-1.10)$ & $106(82)$ & $1.03(1.01-1.05)$ & $1.01(0.99-1.04)$ \\
\hline \multicolumn{7}{|l|}{ T-stage } \\
\hline $\mathrm{T} 1$ & $4(2)$ & 1.00 & 1.00 & $2(1)$ & 1.00 & 1.00 \\
\hline $\mathrm{T} 2$ & 10(3) & $0.65(0.11-3.88)$ & $0.55(0.07-4.50)$ & $10(6)$ & $1.43(0.17-11.85)$ & $0.77(0.08-7.56)$ \\
\hline T3 & $25(9)$ & $0.94(0.20-4.37)$ & $1.49(0.19-11.34)$ & $77(60)$ & $2.95(0.41-21.34)$ & $0.84(0.10-7.08)$ \\
\hline T4 & $22(16)$ & $2.55(0.58-11.15)$ & $1.88(0.19-18.25)$ & $17(15)$ & $3.77(0.50-28.71)$ & $2.12(0.09-48.78)$ \\
\hline \multicolumn{7}{|l|}{$\mathrm{N}$-stage } \\
\hline NO & $33(15)$ & 1.00 & 1.00 & $29(18)$ & 1.00 & 1.00 \\
\hline N1 (metastasis in 1-3 |gl) & $19(9)$ & $1.17(0.51-2.68)$ & $0.55(0.20-1.50)$ & $45(37)$ & $2.41(1.35-4.29)$ & $2.85(1.57-5.17)$ \\
\hline N2 (metastasis in 4 or more lgl) & $9(6)$ & $2.08(0.80-5.37)$ & $8.96(2.47-32.51)$ & $32(27)$ & $2.59(1.40-4.78)$ & $2.45(1.30-4.63)$ \\
\hline \multicolumn{7}{|l|}{ Differentiation grade } \\
\hline Well-moderate & $30(12)$ & 1.00 & 1.00 & $39(24)$ & 1.00 & 1.00 \\
\hline Poor & $31(18)$ & $1.98(0.95-4.11)$ & $2.16(0.77-6.03)$ & $67(58)$ & $2.44(1.50-3.95)$ & $2.13(1.28-3.54)$ \\
\hline \multicolumn{7}{|l|}{ Involved margins, status } \\
\hline RO & $17(4)$ & 1.00 & 1.00 & $6(2)$ & 1.00 & 1.00 \\
\hline$R 1 \& R x$ & $44(26)$ & $2.56(0.89-7.36)$ & $0.46(0.12-1.69)$ & $100(80)$ & $3.49(0.86-14.25)$ & $2.57(0.62-10.60)$ \\
\hline \multicolumn{7}{|l|}{ Lymphatic growth } \\
\hline Absent & $28(7)$ & 1.00 & 1.00 & $32(22)$ & 1.00 & 1.00 \\
\hline Present & $33(23)$ & $3.61(1.55-8.44)$ & $5.85(1.93-17.77)$ & $74(60)$ & $1.51(0.92-2.48)$ & $0.96(0.55-1.70)$ \\
\hline \multicolumn{7}{|l|}{ Vascular growth } \\
\hline Absent & $56(25)$ & 1.00 & 1.00 & $70(47)$ & 1.00 & 1.00 \\
\hline Present & $5(5)$ & $7.78(2.74-22.11)$ & $1.70(0.40-7.31)$ & $36(35)$ & $2.39(1.54-3.72)$ & $2.45(1.54-3.87)$ \\
\hline \multicolumn{7}{|l|}{ Perineural growth } \\
\hline Absent & $42(17)$ & 1.00 & 1.00 & $22(14)$ & 1.00 & 1.00 \\
\hline Present & $19(13)$ & $2.15(1.04-4.44)$ & $3.81(1.55-9.37)$ & $84(68)$ & $1.88(1.05-3.38)$ & $0.92(0.48-1.76)$ \\
\hline \multicolumn{7}{|l|}{ Growth in peripancreatic fat } \\
\hline Absent & $40(14)$ & 1.00 & 1.00 & $22(14)$ & 1.00 & 1.00 \\
\hline Present & $21(16)$ & $3.49(1.68-7.25)$ & $0.75(0.06-9.94)$ & $84(68)$ & $1.80(1.00-3.25)$ & $1.25(0.64-2.43)$ \\
\hline \multicolumn{7}{|l|}{ Adjuvant treatment } \\
\hline No & $43(24)$ & 1.00 & 1.00 & 49 (39) & 1.00 & 1.00 \\
\hline
\end{tabular}


Table 3 Unadjusted and adjusted hazard ratios for death within five years in intestinal and pancreatobiliary type tumours (Continued)

\begin{tabular}{|c|c|c|c|c|c|c|}
\hline Yes & $18(6)$ & $0.60(0.25-1.47)$ & $0.03(0.01-0.16)$ & $57(43)$ & $0.90(0.58-1.39)$ & $0.67(0.43-1.04)$ \\
\hline \multicolumn{7}{|l|}{ PODXL expression } \\
\hline Non-membranous & $49(21)$ & 1.00 & 1.00 & $51(38)$ & 1.00 & 1.00 \\
\hline Membranous & $12(9)$ & $2.32(1.05-5.12)$ & $7.31(2.12-25.16)$ & $55(44)$ & $1.32(0.85-2.03)$ & $1.10(0.67-1.81)$ \\
\hline
\end{tabular}

value. It is however noteworthy that the category of tumours with moderate-strong cytoplasmic staining (score 2) is a somewhat ambiguous group with an intermediate prognosis, undoubtedly harbouring some cases with a prognosis equally poor to cases with membranous PODXL expression. While it is possible that in some of these cases, the presence of membranous expression may be masked by a strong cytoplasmic expression, this category of tumours may also constitute a different biological entity, possibly constituting an "intermediate" between tumours with negative/weak and membranous PODXL expression. In a comparative study on colorectal cancer, membranous expression of the herein used antibody and cytoplasmic expression of an in-house generated antibody were both found to be independent predictors of poor prognosis, and combined use of the antibodies was found to detect a group with an even worse prognosis [28].

Previous studies have demonstrated PODXL to be a functional ligand of E- and L- selectins in pancreatic cancer suggesting that its expression may promote haemotogenic spread of metastases by facilitating binding of circulating tumour cells to selectin-expressing host cells [22]. These findings further support the theory of PODXL overexpression being associated with more aggressive tumours [22]. Moreover, similar to the situation in colorectal $[14,15,16]$ and urinary bladder [19] cancer, PODXL expression was observed predominantly on the invasive tumour front, also suggesting its importance in the metastatic spread of the disease. Of note, in the study on bladder cancer, the herein used polyclonal antibody was compared with two other monoclonal antibodies, all showing $100 \%$ concordance regarding the detection of membranous PODXL expression, whereas the degree of cytoplasmic expression detected by the monoclonal antibodies was substantially weaker [19].

Our results are derived from TMA-based analyses on retrospectively collected tumour samples. Of note, the TMA-technique was also used in the study by Dallas et al. [22]. For characterization of key molecular alterations and expression of investigative biomarkers in tumours from large patient cohorts, whether retrospectively or prospectively defined, the TMA-technology is essential [29].
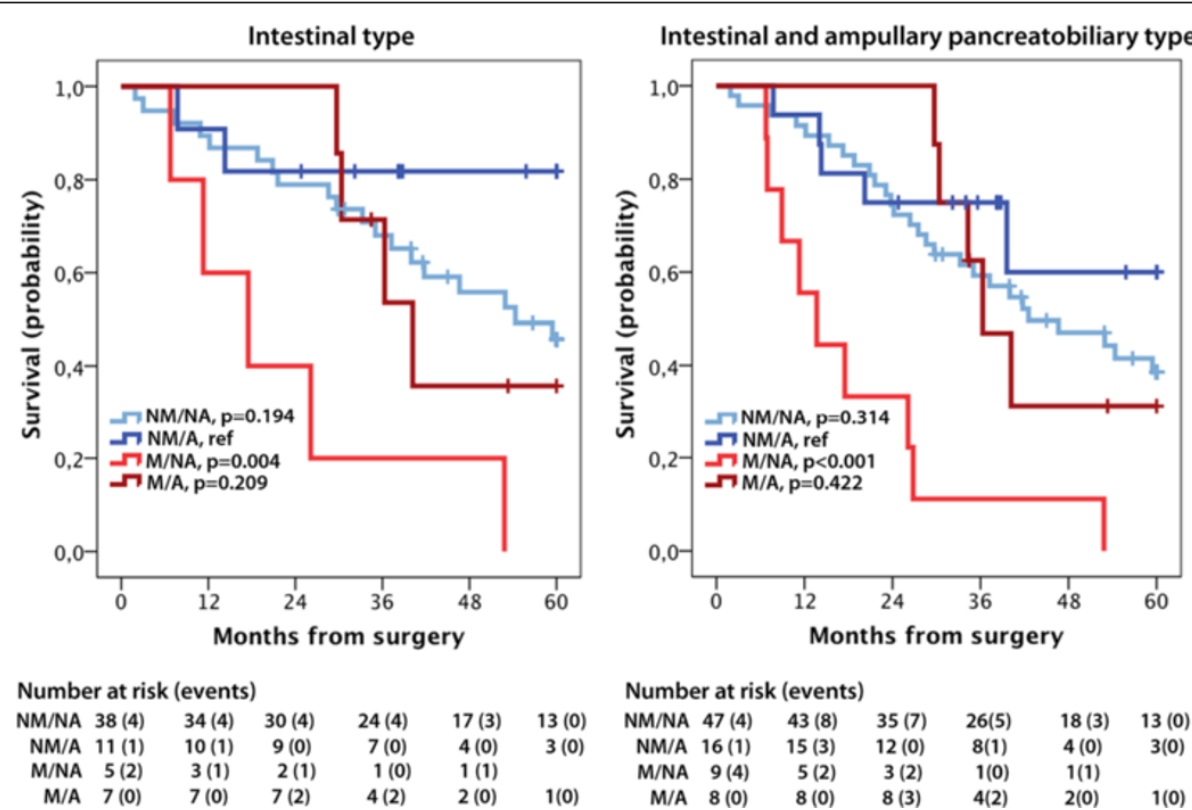

Number at risk (events)

$\begin{array}{rrrrrrr}\text { NM/NA } & 47(4) & 43(8) & 35(7) & 26(5) & 18(3) & 13(0) \\ \text { NM/A } & 16(1) & 15(3) & 12(0) & 8(1) & 4(0) & 3(0) \\ \text { M/NA } & 9(4) & 5(2) & 3(2) & 1(0) & 1(1) & \\ \text { M/A } & 8(0) & 8(0) & 8(3) & 4(2) & 2(0) & 1(0)\end{array}$

Fig. 4 Survival according to PODXL expression and adjuvant chemotherapy. Kaplan-Meier estimates of 5-year overall survival in combined strata of membranous (M)/ non-membranous (NM) PODXL expression and adjuvant (A) /no adjuvant (NA) chemotherapy in patients with intestinal type tumours and intestinal type + ampullary pancreatobiliary type tumours, respectively. $\mathrm{NM}=$ non-membranous, $\mathrm{M}=$ membranous PODXL expression 
However, some limitations related to the TMA-technique must be considered, most importantly its ability to accurately reflect the expression of heterogeneously expressed markers. To compensate for this one needs to ensure that tumour cores are sampled from different regions of the tumour. In the present study, the cores from the primary tumour were, whenever possible, obtained from different donor blocks, and different lymph nodes were sampled in cases with more than one metastatic node.

\section{Conclusions}

Membranous expression of PODXL is significantly higher in pancreatobiliary type as compared with intestinal type periampullary adenocarcinomas and an independent factor of poor prognosis in the latter. The herein presented results also indicate a beneficial effect of adjuvant chemotherapy on intestinal type tumours with membranous PODXL expression, suggesting the potential utility of PODXL as a biomarker for improved treatment stratification of these patients.

\section{Additional files}

Additional file 1: Cox proportional hazards analysis of the impact of PODXL expression on overall survival according to adjuvant treatment intestinal-type and intestinal-type + ampullary pancreatobiliary-type adenocarcinomas.

Additional file 2: Survival according to PODXL score. Kaplan-Meier estimates of recurrence free survival and 5 -year overall survival, respectively, in $(A, B)$ the entire cohort, $(C, D)$ patients with intestinal type tumours and $(E, F)$ patients with pancreatobiliary type tumours. Score $0=$ negative staining, score 1 = weak cytoplasmic positivity in any proportion of cells, score 2: moderate-strong cytoplasmic positivity in any proportion of cells, score 3: distinct membranous positivity in $<=50 \%$ of cells and score $4=$ distinct membranous positivity in $>50 \%$ of cells.

\section{Abbreviations}

PODXL: Podocalyxin-like protein 1; PB-type: Pancreatobiliary type adenocarcinoma; I-type: Intestinal type adenocarcinoma; TMA: Tissue microarray; OS: Overall survival; RFS: Recurrence free survival; HR: Hazard ratio; Cl: Confidence interval.

\section{Competing interests}

The authors declare that they have no competing interests.

\section{Authors' contributions}

$\mathrm{MH}$ collected clinical data, annotated the immunohistochemical staining, performed the statistical analyses and drafted the manuscript. JEL collected clinicopathological data, assisted with TMA construction and helped draft the manuscript. BN constructed the tissue microarray and performed the $\mathrm{IHC}$ stainings. $\mathrm{KJ}$ conceived the study, evaluated the immunohistochemical staining and helped draft the manuscript. JEB collected clinical data, conceived the study and helped draft the manuscript. All authors read and approved the final manuscript.

\section{Acknowledgments}

This study was supported by grants from the Knut and Alice Wallenberg Foundation, the Swedish Cancer Society, the Gunnar Nilsson Cancer Foundation, the Swedish Government Grant for Clinical Research, Lund University Faculty of Medicine and University Hospital Research Grants.

Received: 29 January 2015 Accepted: 18 May 2015

Published online: 30 May 2015

\section{References}

1. C.P, SBWW. World Cancer Report 2014. In: Bernard W, editor. World Cancer Report 2014. Stewart CPW: IARC; 2014

2. Heinemann V, Boeck S, Hinke A, Labianca R, Louvet C. Meta-analysis of randomized trials: evaluation of benefit from gemcitabine-based combination chemotherapy applied in advanced pancreatic cancer. BMC Cancer. 2008;8:82.

3. Jemal A, Siegel R, Ward E, Hao Y, Xu J, Thun MJ. Cancer statistics, 2009. CA Cancer J Clin. 2009:59(4):225-49.

4. Sultana A, Tudur Smith C, Cunningham D, Starling N, Neoptolemos JP, Ghaneh P. Meta-analyses of chemotherapy for locally advanced and metastatic pancreatic cancer: results of secondary end points analyses. Br J Cancer. 2008;99(1):6-13.

5. Herreros-Villanueva M, Hijona E, Cosme A, Bujanda L. Adjuvant and neoadjuvant treatment in pancreatic cancer. World J Gastroenterol. 2012;18(14):1565-72.

6. Westgaard A, Tafjord S, Farstad IN, Cvancarova M, Eide TJ, Mathisen O, et al. Pancreatobiliary versus intestinal histologic type of differentiation is an independent prognostic factor in resected periampullary adenocarcinoma. BMC Cancer. 2008;8:170.

7. Bronsert P, Kohler I, Werner M, Makowiec F, Kuesters S, Hoeppner J, et al. Intestinal-type of differentiation predicts favourable overall survival: confirmatory clinicopathological analysis of 198 periampullary adenocarcinomas of pancreatic, biliary, ampullary and duodenal origin. BMC Cancer. 2013;13:428.

8. Kerjaschki D, Sharkey DJ, Farquhar MG. Identification and characterization of podocalyxin-the major sialoprotein of the renal glomerular epithelial cell. J Cell Biol. 1984;98(4):1591-6.

9. Doyonnas R, Kershaw DB, Duhme C, Merkens H, Chelliah S, Graf T, et al. Anuria, omphalocele, and perinatal lethality in mice lacking the CD34-related protein podocalyxin. J Exp Med. 2001;194(1):13-27.

10. Horvat R, Hovorka A, Dekan G, Poczewski H, Kerjaschki D. Endothelial cell membranes contain podocalyxin-the major sialoprotein of visceral glomerular epithelial cells. J Cell Biol. 1986;102(2):484-91.

11. Doyonnas R, Nielsen JS, Chelliah S, Drew E, Hara T, Miyajima A, et al. Podocalyxin is a CD34-related marker of murine hematopoietic stem cells and embryonic erythroid cells. Blood. 2005;105(11):4170-8.

12. McNagny KM, Pettersson I, Rossi F, Flamme I, Shevchenko A, Mann M, et al. Thrombomucin, a novel cell surface protein that defines thrombocytes and multipotent hematopoietic progenitors. J Cell Biol. 1997;138(6):1395-407.

13. Somasiri A, Nielsen JS, Makretsov N, McCoy ML, Prentice L, Gilks CB, et al. Overexpression of the anti-adhesin podocalyxin is an independent predictor of breast cancer progression. Cancer Res. 2004;64(15):5068-73.

14. Larsson A, Johansson ME, Wangefjord S, Gaber A, Nodin B, Kucharzewska P, et al. Overexpression of podocalyxin-like protein is an independent factor of poor prognosis in colorectal cancer. Br J Cancer. 2011;105(5):666-72.

15. Larsson AH, Fridberg M, Gaber A, Nodin B, Leveen P, Jonsson GB, et al. Validation of podocalyxin-like protein as a biomarker of poor prognosis in colorectal cancer. BMC Cancer. 2012;12(1):282.

16. Larsson AH, Nodin B, Syk I, Palmquist I, Uhlen M, Eberhard J, et al. Podocalyxinlike protein expression in primary colorectal cancer and synchronous lymph node metastases. Diagn Pathol. 2013;8:109.

17. Kaprio T, Fermer C, Hagstrom J, Mustonen H, Bockelman C, Nilsson O, et al. Podocalyxin is a marker of poor prognosis in colorectal cancer. BMC Cancer. 2014:14:493.

18. Cipollone JA, Graves ML, Kobel M, Kalloger SE, Poon T, Gilks CB, et al. The antiadhesive mucin podocalyxin may help initiate the transperitoneal metastasis of high grade serous ovarian carcinoma. Clin Exp Metastasis. 2012;29(3):239-52.

19. Boman $\mathrm{K}$, Larsson AH, Segersten U, Kuteeva E, Johannesson H, Nodin B, et al. Membranous expression of podocalyxin-like protein is an independent factor of poor prognosis in urothelial bladder cancer. Br J Cancer. 2013;108(11):2321-8.

20. Binder ZA, Siu IM, Eberhart CG, Ap Rhys C, Bai RY, Staedtke V, et al. Podocalyxinlike protein is expressed in glioblastoma multiforme stem-like cells and is associated with poor outcome. PLoS One. 2013;8(10):e75945.

21. Ney JT, Zhou H, Sipos B, Buttner R, Chen X, Kloppel G, et al. Podocalyxin-like protein 1 expression is useful to differentiate pancreatic ductal adenocarcinomas from adenocarcinomas of the biliary and gastrointestinal tracts. Hum Pathol. 2007:38(2):359-64.

22. Dallas MR, Chen SH, Streppel MM, Sharma S, Maitra A, Konstantopoulos K. Sialofucosylated podocalyxin is a functional $\mathrm{E}$ - and $\mathrm{L}$-selectin ligand expressed by metastatic pancreatic cancer cells. Am J Physiol Cell Physiol. 2012;303(6):C616-24.

23. Elebro J, Jirstrom K. Use of a standardized diagnostic approach improves the prognostic information of histopathologic factors in pancreatic and periampullary adenocarcinoma. Diagn Pathol. 2014;9(1):80. 
24. Fristedt R, Elebro J, Gaber A, Jonsson L, Heby M, Yudina Y, et al. Reduced expression of the polymeric immunoglobulin receptor in pancreatic and periampullary adenocarcinoma signifies tumour progression and poor prognosis. PLoS One. 2014;9(11):e112728.

25. Elebro J, Heby M, Gaber A, Nodin B, Jonsson L, Fristedt R, et al. Prognostic and treatment predictive significance of SATB1 and SATB2 expression in pancreatic and periampullary adenocarcinoma. J Transl Med. 2014;12(1):289.

26. Cheung $\mathrm{HH}$, Davis AJ, Lee $T L$, Pang AL, Nagrani S, Rennert OM, et al. Methylation of an intronic region regulates miR-199a in testicular tumor malignancy. Oncogene. 2011;30(31):3404-15.

27. Snyder KA, Hughes MR, Hedberg B, Brandon J, Hernaez DC, Bergqvist $P$, et al. Podocalyxin enhances breast tumor growth and metastasis and is a target for monoclonal antibody therapy. Breast Cancer Res. 2015;17(1):46.

28. Kaprio T, Hagstrom J, Fermer C, Mustonen H, Bockelman C, Nilsson O, et al. A comparative study of two PODXL antibodies in 840 colorectal cancer patients. BMC Cancer. 2014;14:494.

29. Torhorst J, Bucher C, Kononen J, Haas P, Zuber M, Kochli OR, et al. Tissue microarrays for rapid linking of molecular changes to clinical endpoints. Am J Pathol. 2001;159(6):2249-56.

\section{Submit your next manuscript to BioMed Central and take full advantage of:}

- Convenient online submission

- Thorough peer review

- No space constraints or color figure charges

- Immediate publication on acceptance

- Inclusion in PubMed, CAS, Scopus and Google Scholar

- Research which is freely available for redistribution 